\section{Justificativas para uso de suplemento em recém-nascidos de baixo risco de um Hospital Amigo da Criança}

\author{
Justifications for formula supplementation in \\ low-risk newborns at a Baby-Friendly Hospital
}

\footnotetext{
${ }_{1}$ Universidade do Grande Rio, Duque de Caxias, Brasil. 2 Hospital Maternidade Carmela Dutra, Secretaria Municipal de Saúde do Rio de Janeiro, Rio de Janeiro, Brasil.

3 Instituto de Saúde da Comunidade, Universidade Federal Fluminense, Niterói Brasil.

4 Instituto Fernandes

Figueira, Fundação Oswaldo Cruz, Rio de Janeiro, Brasil.

Correspondência V. M. Fonseca

Departamento de Epidemiologia e Bioestatística, Instituto de Saúde da Comunidade, Universidade Federal Fluminense.

Estrada Caetano Monteiro 2301, Niterói, RJ 24320-570, Brasil. vaniamf@oi.com.br
}

\begin{abstract}
The Baby-Friendly Hospital Initiative recommends not giving newborn infants any food or drink other than breast milk unless medically indicated. This study investigated the prevalence and alleged reasons for giving formula supplementation to rooming-in newborns at a Baby-Friendly Hospital. Participants were 300 formula-supplemented, exclusively rooming-in newborns at a Baby-Friendly Hospital in Rio de Janeiro, Brazil. Reasons for formula supplementation were classified as acceptable or unacceptable in accordance with the WHO/UNICEF Baby-Friendly Hospital Initiative guidelines. A supplementation prevalence of $33.3 \%$ was found. The main allegations were: hypogalactial agalactia (36.8\%), conditions involving risk of hypoglicemia (21.1\%), cesarean section (7.9\%), stomatognathic system-related conditions (7.4\%), maternal conditions (6.3\%), and absence of maternal HIV serology (4.5\%). Cesarean section was associated with a higher risk of supplementation ( $R P=2.1$; 95\%CI: 1.77-2.55) as compared to vaginal delivery. Supplementation prevalence was high, and only 9\% of the allegations were justified.
\end{abstract}

Supplementary Feeding; Breast Feeding; Newborn Infant
Cynthia de Almeida Brandão Meirelles 1,2 Maria Inês do Couto Oliveira ${ }^{3}$ Rosane Reis de Mello 4 Maria Angélica Bonfim Varela 1,2 Vânia de Matos Fonseca 3,4

\section{Introdução}

As vantagens do aleitamento materno e seu papel na redução da morbimortalidade infantil já são bem conhecidos 1 .

O Programa Nacional de Incentivo ao Aleitamento Materno foi criado em 1981 com a proposta de aumentar os índices de aleitamento materno em nosso país. Nos anos 90, o Brasil assinou a Declaração de Innocenti ${ }^{2}$, incorporando a Iniciativa Hospital Amigo da Criança 3 às atividades do Programa Nacional de Incentivo ao Aleitamento Materno.

O estabelecimento público ou privado ao cumprir "os dez passos para o sucesso do aleitamento materno" 3 da Iniciativa Hospital Amigo da Criança é credenciado como Hospital Amigo da Criança. Segundo dados do Fundo das Nações Unidas para a Infância (UNICEF), o Brasil possui um total de 335 Hospitais Amigos da Criança, dos quais 72 estão na Região Sudeste, sendo 15 no Estado do Rio de Janeiro (UNICEF; http://www. unicef.org/brazil/ihac.htm, acessado em 03/ Mar/2007).

Dos dez passos para o sucesso do aleitamento materno da Iniciativa Hospital Amigo da Criança, o passo 6: "não dar a recém-nascidos nenhum outro alimento ou bebida além do leite materno, a não ser que haja uma indicação médica" 3 (p. 12), é o foco deste estudo. Os motivos médicos listados na recomendação internacional como aceitáveis para a utilização de alimen- 
tos suplementares ou substitutos são de exceção: recém-nascidos de muito baixo peso $(<1.500 \mathrm{~g})$, prematuros com menos de 32 semanas de idade gestacional, os que não conseguem ganhar peso ou manter o grau de hidratação apenas com leite materno, aqueles cuja mãe apresente doença grave ou esteja em uso de medicação contra-indicada durante a amamentação, e os com erro inato do metabolismo ${ }^{3}$. O Ministério da Saúde acrescenta ainda os filhos de mães com sorologia anti-HIV positiva 4.

A suplementação à amamentação pode ser necessária devido à hipoglicemia, em recémnascidos de baixo peso $(<2.500 \mathrm{~g})$, por conta da baixa reserva de glicogênio hepático, assim como nos macrossômicos (> 4.000g) e filhos de mães diabéticas pelo hiperinsulinismo. Logo, em recém-nascidos de baixo risco, como são os que se encontram em alojamento conjunto, a prevalência de suplementação deveria ser baixa. Entretanto, ela ocorre em muitas situações que não são justificáveis pela Iniciativa Hospital Amigo da Criança 5,6.

O uso de substitutos do leite materno nas maternidades pode encorajar as mães a fazerem o mesmo ao retornar para casa, acreditando serem incapazes de produzir leite suficiente. Além disso, essa substituição diminui a freqüência e a efetividade da sucção pelo bebê, reduzindo a quantidade de leite removido das mamas, retardando ou diminuindo a produção e o volume de leite ${ }^{7}$.

A presença de fatores de risco associados a dificuldades na amamentação aumenta a possibilidade de uso de suplemento. As mães submetidas a parto cesáreo, de gemelares, as com intercorrências clínicas, as adolescentes, as primíparas e as com problemas mamários, como o mamilo invertido ou pseudo-invertido necessitam de maior auxílio nas mamadas para que eventuais problemas com a amamentação sejam prevenidos ou minimizados 8 . O desconhecimento materno sobre a amamentação é outro fator associado ao aumento da freqüência do uso de suplemento. Quanto mais informação a mãe tiver, menor a chance de o recém-nascido usar suplemento ${ }^{9}$.

Fora das condições previstas na Iniciativa Hospital Amigo da Criança, que motivos levariam à indicação do suplemento em recém-nascidos de baixo risco? Que situações seriam de maior risco para sua ocorrência? Para responder a essas questões, o Hospital Maternidade Carmela Dutra, da Secretaria Municipal de Saúde do Rio de Janeiro (SMS-RJ), foi escolhido como cenário para a realização deste estudo, pelo fato de possuir o título Hospital Amigo da Criança e ser a segunda maternidade em volume de partos do município (6,6\% do total de nascimentos em 2005) (Sistema de Informações sobre Nascidos Vivos; http://www.datasus.gov.br, acessado em 12/Fev/2008).

O presente estudo teve como objetivo verificar a prevalência de uso de suplemento à amamentação em recém-nascidos internados exclusivamente em alojamento conjunto, identificando se as justificativas alegadas para seu uso foram aceitáveis ou não pela Iniciativa Hospital Amigo da Criança.

\section{Material e métodos}

Trata-se de um estudo transversal, realizado em um Hospital Amigo da Criança do Município do Rio de Janeiro.

O cálculo amostral dos recém-natos foi feito com base em dados de um trabalho de monografia de término de curso de Medicina realizado na mesma maternidade em 2004, que obteve uma prevalência de uso de suplemento de $31 \% 10$. Para um poder de estudo de $99 \%$ e um intervalo de 95\% de confiança (IC95\%) seria necessária uma amostra de 254 recém- nascidos. Segundo a demanda média do ano de 2005 do hospital, estimou-se que esse número de recém-natos seria alcançado em quarenta dias. A pesquisa transcorreu no período de 20 de março a 29 de abril de 2006, chegando-se a 300 recém-nascidos que usaram suplemento dentre os que permaneceram exclusivamente em alojamento conjunto. Foram excluídos os recém-natos da unidade de terapia intensiva (UTI) e intermediária (UI) por não se caracterizarem como baixo risco. Uma ficha de solicitação de suplemento ao Serviço de Nutrição foi criada especialmente para este estudo, da qual foram resgatadas as justificativas alegadas para o uso de suplemento. As características da população foram obtidas dos prontuários, da Declaração de Nascido Vivo, dos livros de registro de nascimentos e de movimentação de pacientes do alojamento conjunto, e do censo estatístico diário do Serviço de Nutrição.

As justificativas alegadas foram listadas de diferentes formas: (1) no total de vezes em que foram citadas em todas as solicitações realizadas, sendo agrupadas conforme suas semelhanças e classificadas em aceitáveis e não aceitáveis pela Iniciativa Hospital Amigo da Criança e (2) considerando somente a primeira justificativa alegada ao ser indicado pela primeira vez o uso de suplemento, apresentando-as por faixas de idade do recém-nascido (menos de 1 hora de vida, de 1-12 horas, e mais de 12 até 96 horas). As 49 alegações encontradas foram agrupadas em 39, conforme suas semelhanças. Destas, seis foram 
condizentes com cinco motivos médicos aceitáveis para indicação de suplementação pela Iniciativa Hospital Amigo da Criança: (1) hipertermia, diurese diminuída e icterícia (fototerapia) enquadravam-se como "recém-nascido com perda aguda de água”; (2) recuperação nutricional foi relacionada a "recém-nascido que não ganha peso através da amamentação"; (3) hipoglicemia, glicemia capilar baixa e glicemia capilar limítrofe estavam de acordo com "recém-nascido que requer terapia para hipoglicemia”; (4) mãe sedada e anestesia geral foram alegações consideradas como um tipo e mãe com problemas psiquiátricos outro, sendo ambos os tipos considerados como "recém-nascido cuja mãe apresenta doença grave"; (5) mãe HIV positivo e Protocolo $n^{o}$. 07611 foram agrupadas como "recém-nascido filho de mãe com sorologia positiva anti-HIV". As justificativas prematuridade e baixo peso não foram consideradas como aceitáveis porque todos os recém-nascidos da amostra tinham idade gestacional acima de 32 semanas e peso maior que $1.500 \mathrm{~g}$.

Para o cálculo amostral do número de mães entrevistadas foi utilizada fórmula para populações finitas com estimação da freqüência esperada sobre ter recebido orientação quanto à importância e manejo do aleitamento materno de $50 \%$ e alfa $=0,05$ e IC95\%, perfazendo um total de 141 mães, o que correspondeu a cerca da metade das mães cujos bebês tiveram prescrição de suplemento no período do estudo. A seleção dessas mães foi feita por meio da ordenação alfabética diária do nome das mães de todos os recém-nascidos com prescrição de suplemento no dia, alternando-se a escolha entre as de numeração par e as de numeração ímpar a cada semana. Obteve-se 143 mulheres, visto que alguns bebês eram gemelares. As entrevistas foram realizadas no alojamento conjunto, visando a esclarecer se as mães tinham ou não recebido orientação quanto à amamentação durante o pré-natal, parto e puerpério, e como apreenderam as informações. As perguntas foram adaptadas dos questionários validados de avaliação global de Hospital Amigo da Criança ${ }^{4}$ e da Iniciativa Unidade Básica Amiga da Amamentação ${ }^{12}$. As respostas às perguntas abertas foram registradas por escrito por entrevistadores treinados, e posteriormente classificadas por uma neonatologista da equipe de pesquisa como "adequadas" ou "inadequadas", segundo critérios adotados pelo programa de avaliação da Iniciativa Hospital Amigo da Criança 4.

As análises estatísticas foram feitas com a utilização do Epi Info 2000 (Centers for Disease Control and Prevention, Atlanta, Estados Unidos). As comparações foram realizadas usando-se o teste qui-quadrado e razão de prevalência (RP). O ní- vel de significância estatística adotado foi o $\mathrm{p}<$ 0,05. O estudo foi aprovado pelo Comitê de Ética em Pesquisa da SMS-RJ, tendo as mulheres envolvidas recebido explicação sobre o objetivo do trabalho e assinado termo de consentimento.

\section{Resultados}

Em março de 2006 ocorreram 693 partos, sendo 454 vaginais (65,5\%) e 239 cesáreos (34,5\%) e, em abril, 760 partos, com 485 vaginais $(63,8 \%)$ e 275 cesáreos $(36,2 \%)$.

No período do estudo nasceram 974 crianças no Hospital Maternidade Carmela Dutra. Destas, 72 estiveram na UTI ou UI neonatal, não sendo elegíveis para o estudo. Portanto, 902 bebês permaneceram exclusivamente em alojamento conjunto, sendo prescrito suplemento para 300 deles (33,3\%).

A Tabela 1 mostra que a maioria $(68 \%)$ das mulheres apresentava idade entre 20-34 anos, $88,2 \%$ tinham entre $4-11$ anos de estudo e mais da metade fez sete ou mais consultas de pré-natal (55\%), observando-se que o pré-natal foi realizado em diferentes instituições.

A média da idade gestacional foi de 38 semanas e 6 dias (desvio-padrão - DP = 1 semana e 4 dias), variando de 32 semanas a 42 semanas e 1 dia. A média do peso ao nascer foi de $3.246 \mathrm{~g}$ (DP $=553,6 \mathrm{~g}$ ), sendo o peso mínimo $1.880 \mathrm{~g}$ e o máximo $4.970 \mathrm{~g}$. No total de 902 recém-nascidos de alojamento conjunto, 581 foram vaginais (64,4\%) e 321 cesáreos (35,6\%), conferindo uma $\mathrm{RP}=2,1$ (IC95\%: 1,77-2,55) para prescrição de suplemento nos recém-nascidos de parto cesáreo em relação aos de parto vaginal. Dentre os 300 recém-nascidos que receberam prescrição de suplemento, 158 (52,6\%) nasceram no período de 07:01 às 19:00 horas e $142(47,4 \%)$ entre 19:01 e 07:00 horas. Usaram suplemento por um dia 161 (53,7\%) bebês; 84 (28\%) durante dois dias e $35(11,7 \%)$ por três dias. Os 20 restantes $(6,7 \%)$ usaram entre 4 e 9 dias (dados não apresentados em tabela).

Para os 300 recém-nascidos foram realizadas 529 solicitações de suplemento, com uma média de 1,8 por bebê. Não houve justificativa registrada em 143 delas (27\% das solicitações), feitas para um grupo de 45 recém-nascidos (15\% da amostra estudada). Nas 386 (73\%) solicitações restantes, feitas para os demais 255 recém-nascidos, foram contadas 606 alegações para o uso de suplemento, visto que a solicitação comportava uma ou mais alegações. Destas 606 alegações, $55(9 \%)$ eram aceitáveis pela Iniciativa Hospital Amigo da Criança e estavam justificando o uso de suplemento em 35 bebês (11,6\% da amostra). 
Tabela 1

Características maternas e dos recém-nascidos com prescrição de suplemento no alojamento conjunto do Hospital Maternidade Carmela Dutra, Rio de Janeiro, Brasil, 2006.

\begin{tabular}{|c|c|c|}
\hline \multirow[t]{2}{*}{ Características } & \multicolumn{2}{|c|}{ Freqüência } \\
\hline & $\mathrm{n}$ & $\%$ \\
\hline \multicolumn{3}{|c|}{ Idade materna (anos) [N = 300] } \\
\hline$<20$ & 55 & 18,3 \\
\hline $20-34$ & 204 & 68,0 \\
\hline$\geq 35$ & 41 & 13,7 \\
\hline \multicolumn{3}{|c|}{ Escolaridade materna (anos) [N = 297] } \\
\hline Nenhuma & 2 & 0,7 \\
\hline $1-3$ & 7 & 2,3 \\
\hline $4-7$ & 130 & 43,8 \\
\hline $8-11$ & 132 & 44,4 \\
\hline$\geq 12$ & 26 & 8,8 \\
\hline \multicolumn{3}{|c|}{ Tipo de parto [ $\mathrm{N}=300]$} \\
\hline Vaginal & 138 & 46,0 \\
\hline Cesáreo & 162 & 54,0 \\
\hline \multicolumn{3}{|c|}{ Número consultas pré-natal [N = 298] } \\
\hline Nenhuma & 8 & 2,7 \\
\hline $1-3$ & 27 & 9,1 \\
\hline $4-6$ & 99 & 33,2 \\
\hline$\geq 7$ & 164 & 55,0 \\
\hline \multicolumn{3}{|c|}{ Tipo de gravidez [N = 300] } \\
\hline Única & 275 & 91,7 \\
\hline Gemelar & 25 & 8,3 \\
\hline \multicolumn{3}{|c|}{ Idade gestacional (semanas) [N = 300] } \\
\hline $32-37$ & 27 & 9,0 \\
\hline$\geq 37$ e $<42$ & 270 & 90,0 \\
\hline$\geq 42$ & 3 & 1,0 \\
\hline \multicolumn{3}{|c|}{ Peso ao nascer (gramas) [N = 300] } \\
\hline $1.500-2.500$ & 28 & 9,3 \\
\hline $2.501-4.000$ & 241 & 80,3 \\
\hline$\geq 4.001$ & 31 & 10,3 \\
\hline \multicolumn{3}{|l|}{ Sexo $[N=300]$} \\
\hline Masculino & 155 & 51,6 \\
\hline Feminino & 145 & 48,4 \\
\hline \multicolumn{3}{|c|}{ Classificação Lubchenco * [N = 300] } \\
\hline $\mathrm{AIG}$ & 240 & 80,0 \\
\hline PIG & 11 & 3,7 \\
\hline GIG & 49 & 16,3 \\
\hline \multicolumn{3}{|c|}{ Classificação Alexander ** [N = 300] } \\
\hline AIG & 226 & 75,3 \\
\hline PIG & 47 & 15,7 \\
\hline GIG & 27 & 9,0 \\
\hline
\end{tabular}

AIG: adequado para a idade gestacional; PIG: pequeno para a idade gestacional; GIG: grande para a idade gestacional.

* Lubchenco et al. 13;

** Alexander et al. 14 .
As outras 549 alegações (91\%) eram não aceitáveis pela Iniciativa Hospital Amigo da Criança e foram encontradas como justificativas para o uso de suplemento em 220 neonatos $(73,4 \%$ da amostra).

As 606 justificativas alegadas registradas em 386 solicitações foram agrupadas conforme suas semelhanças, sendo apresentadas na Tabela 2. As mais prevalentes foram "hipogalactia", seguida de "agalactia”, "parto cesáreo", "recém-nascido grande para a idade gestacional (GIG)", "dificuldade de sucção" e "mãe sem resultado de anti-HIV", as quais, juntas, foram citadas 374 vezes $(61,8 \%)$, sendo todas não aceitáveis pela Iniciativa Hospital Amigo da Criança. A justificativa aceitável mais prevalente foi "hipoglicemia" $(3,6 \%)$.

Ao considerar-se a primeira solicitação de uso de suplemento, a média de idade do recémnascido foi de 9,5 horas (DP = 14,3), com mediana de 3 horas, variando de 0 a 96 horas de vida. A Tabela 3 apresenta essas justificativas segundo o tempo de vida do recém-nascido ao ser feito o primeiro pedido de suplemento. Nota-se que em 127 (42,3\%) o suplemento foi iniciado com menos de 1 hora de vida; em 94 vezes $(31,3 \%)$ entre uma e 12 horas; e em 79 ocasiões (26,3\%) entre 13 e 96 horas de vida. Na primeira hora de vida, a RP para o uso de suplemento foi de 1,9 (IC95\%: 1,42-2,60) nos nascidos de parto cesáreo em relação aos de parto vaginal. Em 72 (24\%) destas, nas 300 solicitações iniciais nenhuma justificativa foi mencionada. Em apenas 9 (4\%) das demais, 228 solicitações tinham alguma alegação aceitável pela Iniciativa Hospital Amigo da Criança.

Somente cinco recém-nascidos tiveram solicitação de uso de suplemento com mais de 48 horas de vida, sendo quatro sem justificativa registrada e uma com a alegação "hipogalactia”. Do total de 112 justificativas "hipogalactia/agalactia”, 96 (85,7\%) incidiram em bebês com menos de 24 horas de vida.

No total da amostra, 25 recém-nascidos eram gemelares, com RP para o uso de suplemento com menos de 1 hora de vida de 2,0 (IC95\%: 1,61-2,63) em relação aos não gemelares. Nenhum deles usou suplemento com justificativa aceitável pela Iniciativa Hospital Amigo da Criança. O risco de ter prescrição de suplemento antes da primeira hora de vida ocorreu para os gemelares tanto em parto vaginal $(\mathrm{RP}=3,24$; IC95\%: 2,04-5,13) quanto em parto cesáreo (RP = 1,55; IC95\%:1,17-2,05). A gemelaridade apareceu como fator de risco para uso de suplemento com menos de 1 hora de vida tanto para prematuros $(\mathrm{RP}=1,92$; IC95\%: 0,89-4,10) quanto para não prematuros (RP = 2,08; IC95\%: 1,61-2,70) (dados não apresentados em tabela). 
Justificativas fornecidas para o uso de suplemento em recém-nascidos no alojamento conjunto no Hospital Maternidade Carmela Dutra, Rio de Janeiro, Brasil, 2006

\begin{tabular}{|c|c|c|}
\hline \multirow[t]{2}{*}{ Justificativas } & \multicolumn{2}{|c|}{ Freqüência } \\
\hline & $\mathrm{n}$ & $\%$ \\
\hline \multicolumn{3}{|l|}{ Condições relacionadas à produção de leite } \\
\hline Hipogalactia & 160 & 26,40 \\
\hline Agalactia & 63 & 10,40 \\
\hline Apojadura demorada & 2 & 0,33 \\
\hline \multicolumn{3}{|l|}{ Condições relacionadas ao parto } \\
\hline Parto cesáreo/Pós-operatório & 48 & 7,92 \\
\hline Parto fórceps & 2 & 0,33 \\
\hline \multicolumn{3}{|l|}{ Condições relativas à sorologia materna anti-HIV } \\
\hline Mãe sem resultado anti-HIV & 27 & 4,50 \\
\hline Mãe anti-HIV positivo/Protocolo $n^{\circ} .076^{*}$ & 15 & 2,47 \\
\hline \multicolumn{3}{|l|}{ Recém-nascido que requer terapia para hipoglicemia } \\
\hline Hipoglicemia/Glicemia capilar baixa ou limítrofe * & 22 & 3,63 \\
\hline \multicolumn{3}{|l|}{ Condições de risco para hipoglicemia no recém-nascido } \\
\hline Recém-nascido GIG & 42 & 6,93 \\
\hline Gemelar & 23 & 3,80 \\
\hline Prematuro & 20 & 3,30 \\
\hline Mãe diabética & 11 & 1,82 \\
\hline Recém-nascido PIG & 9 & 1,48 \\
\hline Baixo peso & 8 & 1,32 \\
\hline Risco de hipoglicemia & 8 & 1,32 \\
\hline Macrossomia/Peso > 4.000g & 5 & 0,82 \\
\hline Hipoglicemia no dia anterior & 2 & 0,33 \\
\hline \multicolumn{3}{|l|}{ Condições relativas ao sistema estomatognático do recém-nascido } \\
\hline Sucção débil/Dificuldade de sucção & 34 & 5,61 \\
\hline Dificuldade de pega & 11 & 1,82 \\
\hline \multicolumn{3}{|l|}{ Problemas mamilares } \\
\hline Mamilos planos & 10 & 1,65 \\
\hline Mamilos invertidos & 9 & 1,48 \\
\hline Problemas no bico & 2 & 0,33 \\
\hline \multicolumn{3}{|l|}{ Recém-nascido com perda de água } \\
\hline Hipertermia/Diurese diminuída/lcterícia (em fototerapia) * & 8 & 1,32 \\
\hline \multicolumn{3}{|l|}{ Recém-nascido cuja mãe apresenta doença grave } \\
\hline Mãe sedada/Anestesia geral * & 5 & 0,82 \\
\hline Mãe com problemas psiquiátricos * & 3 & 0,49 \\
\hline \multicolumn{3}{|l|}{ Situações relacionadas à condição materna } \\
\hline Mãe "sem condições" ou "com dor" & 20 & 3,30 \\
\hline Dificuldade para amamentar & 7 & 1,15 \\
\hline Primípara & 3 & 0,49 \\
\hline Mãe com bócio & 2 & 0,33 \\
\hline Mãe sem interesse & 2 & 0,33 \\
\hline Mãe com distúrbio emocional & 2 & 0,33 \\
\hline Mãe adolescente & 2 & 0,33 \\
\hline \multicolumn{3}{|l|}{ Outras condições relacionadas ao recém-nascido } \\
\hline Hipoatividade & 2 & 0,33 \\
\hline Recém-nascido sem interesse & 2 & 0,33 \\
\hline Apgar $3 / 8$ & 2 & 0,33 \\
\hline \multicolumn{3}{|c|}{ Recém-nascido que não ganha peso somente através da amamentação } \\
\hline Recém-nascido em recuperação nutricional * & 2 & 0,33 \\
\hline
\end{tabular}

(continua) 


\begin{tabular}{lcc} 
Tabela 2 (continuação) & Freqüência & $\%$ \\
\hline Justificativas & $\mathbf{n}$ & \\
& & 0,82 \\
Outras & 5 & 0,49 \\
"Se necessário" & 3 & 0,49 \\
"Complemento" & 3 & 100,00 \\
"Transcrição de prescrição médica" & 606 & \\
Total & &
\end{tabular}

GIG: grande para a idade gestacional; PIG: pequeno para a idade gestacional.

* Justificativas aceitáveis pela norma da Iniciativa Hospital Amigo da Criança.

Primeira justificativa informada na primeira solicitação de suplemento para o recém-nascido em alojamento conjunto, segundo o tempo de vida, no Hospital Maternidade Carmela Dutra, Rio de Janeiro, Brasil, 2006.

\begin{tabular}{|c|c|c|c|c|}
\hline Primeira justificativa fornecida na primeira solicitação de suplemento & $<1$ hora & 1-12 horas & $12-96$ horas & Total (\%) \\
\hline \multicolumn{5}{|l|}{ Aceitáveis } \\
\hline Hipoglicemia & 0 & 1 & 3 & $4(1,3)$ \\
\hline Mãe com sorologia anti-HIV positiva & 3 & 0 & 0 & $3(1,0)$ \\
\hline Problema psiquiátrico materno & 2 & 0 & 0 & $2(0,7)$ \\
\hline \multicolumn{5}{|l|}{ Não aceitáveis } \\
\hline Hipogalactia/Agalactia & 20 & 49 & 43 & $112(37,3)$ \\
\hline Condições de risco para hipoglicemia no recém-nascido & 19 & 10 & 3 & $32(10,7)$ \\
\hline \multicolumn{5}{|c|}{ (GIG/macrossomia/prematuro/gemelar/mãe diabética/hipoglicemia no dia anterior) } \\
\hline Cesárea/Pós-operatório & 20 & 3 & 1 & $24(8,0)$ \\
\hline Mãe sem resultado de sorologia anti-HIV & 17 & 3 & 1 & $21(7,0)$ \\
\hline \multicolumn{5}{|l|}{ (dificuldade de sucção ou pega/sucção débil) } \\
\hline Problemas mamilares (mamilos invertidos ou planos/problemas no bico) & 2 & 3 & 1 & $6(2,0)$ \\
\hline Situações relacionadas à condição materna & 1 & 3 & 2 & $6(2,0)$ \\
\hline \multicolumn{5}{|l|}{ (mãe sem condições/primípara/dificuldade para amamentar) } \\
\hline Outras & 4 & 2 & 2 & $8(2,6)$ \\
\hline Aceitabilidade ignorada (não preenchida) & 37 & 13 & 22 & $72(24,0)$ \\
\hline Total $(\%)$ & $127(42,3)$ & $94(31,3)$ & $79(26,3)$ & $300(100,0)$ \\
\hline
\end{tabular}

GIG: grande para a idade gestacional.

Nas entrevistas com as mães relativas às orientações recebidas no pré-natal, parto e puerpério, vê-se na Tabela 4 que apenas $51 \%$ das entrevistadas foram informadas quanto ao manejo da amamentação durante o pré-natal e que $35,7 \%$ não receberam ajuda na sala de parto para iniciar a amamentação, e a maioria $(94,1 \%)$ desconhecia o motivo da falta de auxílio.

O parto cesáreo mostrou efeito protetor para receber ajuda para iniciar a amamentação, com $\mathrm{RP}=$ 0,60 (IC95\%: 0,38-0,94) em relação ao parto vaginal. Do grupo de 54 mulheres submetidas à cesariana que receberam ajuda para amamentar no centro cirúrgico, 34 (63\%) tiveram solicitação de suplemento para seus bebês com menos de 1 hora de vida. Não houve registro de justificativa em 11 solicitações; "hipogalactia” ou "agalactia” foram citadas em oito; as condições de risco para hipoglicemia (GIG, prematuro e gemelar) em seis; "parto cesáreo/pós-operatório" em cinco; e, em quatro, outras justificativas. A ausência de orientação no hospital quanto ao modo de colocar o bebê para mamar foi mais prevalente para as mães que tiveram parto cesáreo, quando comparadas às que tiveram parto vaginal $(\mathrm{RP}=$ 2,2; IC95\%: 1,09-4,40) (dados não apresentados em tabela). 
Orientações quanto à amamentação recebidas no período do pré-natal, parto e puerpério pelas mães dos recém-nascidos com suplemento prescrito no alojamento conjunto no Hospital Maternidade Carmela Dutra, Rio de Janeiro, Brasil, 2006.

\begin{tabular}{|c|c|c|c|c|}
\hline Passo & Perguntas & Resposta & $\mathbf{n}$ & $\%$ \\
\hline \multirow[t]{3}{*}{3} & Foi orientada no pré-natal sobre vantagens da amamentação? [N = 143] & Sim & 101 & 70,6 \\
\hline & Foi orientada no pré-natal sobre manejo da amamentação? [N = 143] & $\operatorname{Sim}$ & 73 & 51,0 \\
\hline & Como foi orientada? [N = 73] & Adequada & 58 & 79,5 \\
\hline \multirow[t]{2}{*}{4} & Recebeu ajuda na sala de parto para colocar o bebê para mamar? [N = 143] & $\operatorname{Sim}$ & 92 & 64,3 \\
\hline & Sabe por que não? [N = 51] & Adequada & 3 & 5,9 \\
\hline \multirow[t]{5}{*}{5} & Foi orientada sobre como colocar para mamar? [N = 143] & Sim & 111 & 77,6 \\
\hline & Como foi a orientação? [N = 111] & Adequada & 90 & 81,1 \\
\hline & Foi orientada sobre como tirar leite do peito? [N = 143] & Sim & 60 & 42,0 \\
\hline & Como foi a orientação? [N = 60] & Adequada & 52 & 86,7 \\
\hline & Foi orientada que maior a sucção, maior a produção de leite? [N=143] & $\operatorname{Sim}$ & 125 & 87,5 \\
\hline 8 & Foi orientada para deixar o bebê mamar quando quiser? [N=143] & Sim & 83 & 58,0 \\
\hline \multirow[t]{4}{*}{9} & Recebeu orientação para não dar mamadeira? [N =143] & $\operatorname{Sim}$ & 80 & 55,9 \\
\hline & O que foi falado? [N = 80] & Adequada & 54 & 67,5 \\
\hline & Recebeu orientação para não dar chupeta? [N = 143] & Sim & 47 & 32,9 \\
\hline & O que foi falado? [N = 47] & Adequada & 35 & 74,5 \\
\hline \multirow[t]{5}{*}{6} & Seu bebê recebeu outro leite? [N=143] & $\operatorname{Sim}$ & 125 & 87,4 \\
\hline & Foi informada sobre o motivo? [ $\mathrm{N}=143]$ & Sim & 107 & 74,8 \\
\hline & Sabe dizer qual? [N = 107] & Adequada & 11 & 10,3 \\
\hline & Você acha que o leite faz mal? [N = 143] & $\operatorname{Sim}$ & 45 & 31,5 \\
\hline & Por que você acha que faz mal? [N = 45] & Adequada & 33 & 73,3 \\
\hline \multirow[t]{2}{*}{ Outras } & Foi informada no hospital sobre o tempo de aleitamento materno exclusivo? [ $N=143]$ & Sim & 93 & 65,0 \\
\hline & Você sabe qual é o tempo recomendado? [N = 143] & Adequada & 96 & 67,1 \\
\hline
\end{tabular}

Das mães entrevistadas, $42 \%$ foram orientadas quanto à ordenha manual e, destas, 86,7\% sabiam a técnica adequada. Em relação ao uso de chupeta, 32,9\% relataram ter recebido orientação quanto a não usá-la, das quais apenas $74,5 \%$ sabiam informar uma razão adequada para esta recomendação (Tabela 4). As mães de parto cesáreo foram mais orientadas a não oferecer chupeta, quando comparadas às de parto vaginal $(\mathrm{RP}=$ 0,8; IC95\%: 0,63-0,99).

Apesar de solicitado, alguns bebês não tinham usado suplemento até o momento da entrevista (12,6\%). Das 107 mães que foram informadas sobre o motivo da prescrição do suplemento, somente $10,3 \%$ relataram um motivo aceitável pela Iniciativa Hospital Amigo da Criança.

Não houve diferença estatisticamente significativa para a mãe estar ou não orientada sobre todas as questões abordadas na entrevista, no que diz respeito à idade e escolaridade maternas, assim como ao número de consultas de pré-natal realizadas (dados não apresentados em tabela).

\section{Discussão}

Apesar de se tratar de um Hospital Amigo da Criança e da população estudada ser composta de recém-nascidos de baixo risco, um terço deles teve suplemento prescrito. Os recém-natos que permaneceram exclusivamente em alojamento conjunto caracterizaram-se como de baixo risco pelo fato de a maioria ter nascido a termo, com peso ao nascer entre $2.500 \mathrm{~g}$ e $4.000 \mathrm{~g}$, e adequados para a idade gestacional tanto pela classificação de Lubchenco et al. 13 quanto pela de Alexander et al. 14 .

Gagnon et al. 6, em estudo sobre uso de suplementação por fórmula em recém-nascidos saudáveis nascidos de parto vaginal de uma maternidade no Canadá, encontraram uma taxa de $47,9 \%$, sendo de 8,4 horas a média de idade do recém-nato ao ser prescrito o primeiro suplemento. No presente estudo essa média foi de 9,5 horas, tendo $42 \%$ iniciado com menos de 1 hora de vida. Chama atenção a idade precoce de início de suplemento para bebês de baixo risco, demonstrando uma precipitação na prescrição de suplemento por parte dos profissionais. 
Em cerca de um quarto das solicitações nenhuma justificativa foi informada. A falta de hábito ou não valorização em registrar a indicação do uso de suplemento, a intenção de não expor a indicação inadequada do suplemento, o desestímulo ou insatisfação do profissional na sua atividade ou a sobrecarga de trabalho são explicações possíveis para o grande percentual de não preenchimento.

Do total de justificativas, menos de um décimo delas estava de acordo com os critérios da Iniciativa Hospital Amigo da Criança. A baixa ocorrência de justificativas aceitáveis pela Iniciativa Hospital Amigo da Criança pode estar relacionada a: (1) inadequação entre a condição clínica do recém-nascido e o registro da justificativa; (2) equívoco no diagnóstico; (3) desconhecimento das indicações de uso de suplemento segundo a Iniciativa Hospital Amigo da Criança ou dos malefícios que o uso indevido de suplemento pode acarretar na duração do aleitamento materno; (4) ser reflexo da opinião dos profissionais e das mães em relação à amamentação 6,9.

Em estudo sobre indicação de suplemento, Ekström \& Nissen 15 constataram que a suplementação por razões médicas não teve influência significativa na duração da amamentação, enquanto que, quando o suplemento foi utilizado sem motivos médicos, houve associação com um período menor de aleitamento materno exclusivo e de amamentação.

"Hipogalactia" e "agalactia" responderam por mais de um terço do total de justificativas (Tabela 2). Sabe-se que a produção de leite está intimamente ligada ao estímulo pela sucção do bebê, sendo considerado o mais importante fator para a continuidade do leite produzido 7 . O uso de suplemento provoca uma diminuição da freqüência e da efetividade da sucção, o que reduz a quantidade de leite removido das mamas, retardando a produção ou diminuindo o volume de leite 7 . Logo, tais justificativas não seriam adequadas para prescrição de suplemento, principalmente nas primeiras horas de vida, visto que a quantidade de leite é fisiologicamente pequena, pois ainda não ocorreu a apojadura, esperada entre 48 e 72 horas após o parto. "Hipogalactia" e "agalactia” responderam por cerca da metade das indicações de início de uso de suplemento. Dessa forma, reforça-se a inadequação no diagnóstico da condição clínica apresentada ou no registro da justificativa, principalmente para os bebês com menos de 24 horas de vida, quando a quantidade de leite materno ainda é pouca e a mama precisa do estímulo da sucção, o que não ocorrerá adequadamente se o recém-nascido estiver saciado, devido ao suplemento.
As justificativas vinculadas a condições de risco para hipoglicemia foram responsáveis por $10,7 \%$ das indicações para início de suplemento. Entre os bebês que iniciaram suplemento com menos de 1 hora de vida essas condições responderam por $21,1 \%$ dos casos. Mesmo não sendo razões aceitáveis pela Iniciativa Hospital Amigo da Criança, alguns autores encontram o uso de suplemento associado a condições comumente ligadas a hipoglicemia, como o recém-nascido de baixo peso, os macrossômicos e filhos de mães diabéticas 5,6,16. Os prematuros incluem-se nesse grupo por terem a sucção fisiologicamente fraca, dificuldades na capacidade de deglutir, digerir e absorver 17, por características anatômicas e neurológicas da idade gestacional. A sucção menos eficiente não estimula a mama adequadamente, com conseqüente redução da produção de leite, deixando-os mais susceptíveis à ocorrência de hipoglicemia, levando ao uso de suplemento. Entretanto, no presente estudo não houve diferença significativa em relação ao uso de suplemento na primeira hora entre os não prematuros e os prematuros (nesta amostra, todos acima de 32 semanas). Dentre os gemelares, o risco de ter prescrição antes da primeira hora de vida ocorreu tanto em parto vaginal como no cesáreo. Com menos de 1 hora de vida, os gemelares tiveram mais suplemento prescrito em relação aos não gemelares. As mães de gemelares são mais susceptíveis a dificuldades para amamentar e a associação entre gemelaridade e prematuridade pode ser um fator determinante destas dificuldades 18. Porém, como a gemelaridade foi fator de risco para uso de suplemento com menos de 1 hora de vida tanto para prematuros quanto para não prematuros, parece que possíveis dificuldades para a amamentação em gemelares foram supostas antes que ocorressem. Nesse caso, o uso de suplemento não foi um marcador de dificuldades na amamentação. De todo modo, a razão aceitável pela Iniciativa Hospital Amigo da Criança para usar suplemento seria a hipoglicemia já instalada, e não a possibilidade dela acontecer. Apenas 3,6\% das alegações de uso de suplemento foram por hipoglicemia.

Foi encontrada uma maior prevalência de uso de suplemento em bebês que nasceram de parto cesáreo em relação aos de parto vaginal, resultado semelhante a outros estudos 5,19 . O parto cesáreo foi citado como justificativa 7,9\% das vezes. Como a indicação de início de uso de suplemento foi mais freqüente nos bebês com menos de 1 hora de vida, pode-se supor uma precipitação da prescrição de suplemento antes que surgissem dificuldades. Esses achados podem também refletir uma resistência dos profissionais em possibilitar o contato dessas mães com seus bebês, 
dificuldades na operacionalização deste contato, ou, ainda, um atraso no estabelecimento do alojamento conjunto ${ }^{20}$. Berra et al. 21 encontraram risco significativamente maior do bebê não estar em aleitamento materno exclusivo durante o pós-parto imediato em instituições públicas quando o primeiro alimento foi diferente do leite materno e quando o primeiro contato mãe/bebê atrasou 45 minutos. Sabe-se que o início da sucção com menos de 1 hora de vida influencia positivamente a duração do aleitamento materno 22. Portanto, fica assinalado o descumprimento do passo 4 da Iniciativa Hospital Amigo da Criança, que recomenda ajudar as mães a iniciar o contato pele a pele e a amamentação na primeira meia hora de vida.

Uma alegação não esperada foi "mãe sem resultado de sorologia anti-HIV". O surgimento dessa justificativa não aceitável pela Iniciativa Hospital Amigo da Criança evidencia dificuldades operacionais na realização do teste rápido anti-HIV. Este é um teste de triagem, com sensibilidade alta, para identificação das gestantes com sorologia positiva. É preconizado pelo Ministério da Saúde para que intervenções profiláticas sejam realizadas, reduzindo a probabilidade de transmissão vertical do HIV. Desse modo, o status sorológico materno deve ser conhecido antes do nascimento do bebê e o suplemento só deve ser prescrito em vigência de positividade, pois o aleitamento materno representa um risco adicional de $7 \%$ a $22 \%$ na transmissão do vírus ${ }^{23}$. Esse teste deve ser realizado na admissão, por ser de manejo simples, ao utilizar sangue venoso ou de polpa digital, gerando resultados de fácil interpretação em até trinta minutos, evitando-se o uso desnecessário de suplemento.

As condições relativas ao sistema estomatognático do recém-nascido representaram $7,4 \%$ do total das alegações (Tabela 2), sendo indicação de início de uso de suplemento com uma freqüência de 3,3\% (Tabela 3). A maioria das solicitações com essas justificativas foi feita para bebês entre 1 e 12 horas de vida. Embora não sejam razões aceitáveis pela Iniciativa Hospital Amigo da Criança para prescrição de suplemento, as disfunções motora-orais foram relacionadas a dificuldades na amamentação e associadas ao uso de suplemento no hospital em uma revisão bibliográfica 24 . A pronta identificação e correção dessas disfunções logo nas primeiras mamadas é recomendada para que o padrão incorreto não se estabeleça como um hábito permanente, gerando um desempenho insatisfatório na mamada, culminando com o uso de suplemento e desmame precoce 26 .

A presença de justificativas relacionadas a intercorrências clínicas maternas, primiparida- de, mãe adolescente e problemas mamilares 8 reforçam a afirmação de que as mães com estas condições necessitam de um maior auxílio nas primeiras mamadas para prevenção de eventuais problemas.

Neste estudo não houve influência do número de consultas de pré-natal no uso de suplementação, apesar de saber-se que o desconhecimento materno sobre amamentação pode contribuir para o surgimento de dificuldades na sua prática, levando ao uso indevido de suplemento ${ }^{9}$.

O parto cesáreo apareceu como fator protetor para recebimento de ajuda para amamentar na sala de parto quando comparado ao parto vaginal. Entretanto, quase dois terços dos bebês cujas mães, submetidas a parto cesáreo, asseguraram terem recebido ajuda para amamentar (passo 4), tiveram suplemento prescrito com menos de 1 hora de vida. A principal justificativa para uso de suplemento nesses bebês foi "hipogalactia/ agalactia", seguida das "condições de risco para hipoglicemia” (GIG, prematuro e gemelar) e de "parto cesáreo". Um dos motivos pelo qual o parto cesáreo é comumente associado ao uso de suplemento é o atraso no contato mãe/bebê, o que não ocorreu, pelo fato das mães terem recebido ajuda. Os bebês não teriam motivo para usar suplemento com menos de 1 hora de vida, a não ser por razões médicas aceitáveis pela Iniciativa Hospital Amigo da Criança, o que não foi registrado nas solicitações, supondo-se novamente uma precipitação na prescrição de suplemento. Em contrapartida, não ser orientada no hospital quanto a como colocar seu bebê para mamar (passo 5) foi mais prevalente entre as mães submetidas a parto cesáreo comparadas às de parto vaginal $(R P=2,2)$. Em tese, as pacientes de parto cesáreo, por demandarem um maior cuidado, estariam mais expostas ao contato com a equipe de saúde e, conseqüentemente, a mais orientações e ajuda relativas à mamada. Isso possivelmente indica que o profissional possa ter desviado o foco de atenção da amamentação para os cuidados pós-operatórios.

Apenas $42 \%$ das entrevistadas relataram terem sido orientadas quanto à ordenha manual (passo 5). Esse conhecimento é necessário no caso do bebê não estar exercendo uma sucção adequada para fornecer estímulo à produção de leite, pois, ao promover o esvaziamento da mama, a ordenha contribui para o reflexo neuroendócrino e a atividade secretora láctea 7 . Por outro lado, quando a mama está excessivamente cheia, o bebê não consegue abocanhar a aréola para a sucção adequada, sendo a ordenha útil para facilitar a pega. Em ambas as situações, o conhecimento materno dessa prática ajuda a diminuir o risco de uso de suplemento. 
Em relação ao passo 9, cerca da metade das mães afirmaram ter recebido orientação para não usar mamadeira, dois terços das quais sabiam informar a razão. Por outro lado, apenas um terço disse ter sido orientada a não oferecer chupeta ao seu bebê, três quartos das quais sabiam informar o motivo. O desconhecimento materno sobre essas recomendações deixa espaço para a mãe oferecer mamadeira ou chupeta ao bebê ainda no hospital, hábito que faz parte da nossa cultura. Seu uso pode levar à confusão de bicos, ocasionando disfunção motora-oral, com maior risco de suplementação 25 .

Somente $10,3 \%$ das mães que disseram saber a razão pela qual seu bebê estava usando suplemento informaram uma justificativa aceitável pela Iniciativa Hospital Amigo da Criança. Assim, é possível que a mãe fique com um juízo errôneo da indicação de uso de suplemento, podendo reproduzi-lo inadequadamente após a alta, com a impressão de estar correta em sua conduta. $\mathrm{O}$ uso abusivo de suplemento no hospital reforça a idéia de incapacidade em prover a alimentação do bebê somente com o leite materno, aumentando a possibilidade de suplementação após a alta, comprometendo a duração do aleitamento materno 6

Menos de um terço das mães respondeu acreditar nos malefícios do leite artificial. Campanhas publicitárias maciças realizadas no passado pela indústria de substitutos do leite materno ainda repercutem na percepção de inocuidade das fórmulas 26 , o que parece gerar uma permissividade e tolerância maior quanto a seu uso.

Verificamos que as orientações sobre aleitamento materno recebidas durante o pré-natal, parto e puerpério foram insuficientes (Tabela 4). Os conceitos sobre amamentação sofrem influências do meio (significados e valores culturais de uma comunidade), das condições psíquicas (experiências anteriores e significado de valor do aleitamento materno), das condições biológicas (dor ou enfermidades) e do apoio social (família, trabalho, creches) e interferem na tomada de decisão das pessoas. Portanto, a amamentação é um comportamento humano complexo e a sua vivência é variável. A abordagem das gestantes e mães depende da capacidade do profissional de saúde para lidar com as diferenças, comunicarse e alcançar o objetivo de fazer as orientações tornarem-se efetivas na prática 27 .

A ocorrência de justificativas não aceitáveis pela Iniciativa Hospital Amigo da Criança indica a necessidade de um programa de capacitação para os profissionais de saúde, utilizando metodologias ativas de ensino que permitam melhor apreensão do conhecimento e desenvolvimento de habilidades e atitudes, para a conscientização da magnitude do seu papel na manutenção e efetividade da Iniciativa Hospital Amigo da Criança. 


\section{Resumo}

A Iniciativa Hospital Amigo da Criança preconiza que não se dê a recém-natos nenhum outro alimento ou líquido além do leite materno, a não ser que haja indicação clínica (passo 6). Este estudo teve como objetivo verificar a prevalência e identificar justificativas alegadas para suplementação ao aleitamento materno em recém-nascidos de alojamento conjunto. A amostra foi composta por 300 recém-nascidos de um Hospital Amigo da Criança do Rio de Janeiro, Brasil, que usaram suplemento dentre os que permaneceram exclusivamente em alojamento conjunto. As justificativas alegadas para uso de suplemento foram classificadas como aceitáveis ou não segundo critérios da Iniciativa Hospital Amigo da Criança. A prevalência de uso de suplemento foi de $33,3 \%$. As principais justificativas foram: hipogalactialagalactia (36,8\%), condições de risco para hipoglicemia $(21,1 \%)$, parto cesáreo $(7,9 \%)$, condições relativas ao sistema estomatognático $(7,4 \%)$, condições maternas $(6,3 \%)$ e ausência de resultado de teste rápido anti-HIV (4,5\%). O parto cesáreo esteve associado à maior risco de uso de suplemento $(R P=2,1$; IC95\%: 1,77-2,55) em relação ao parto vaginal. A prevalência do uso de suplemento foi elevada, sendo 9\% das justificativas alegadas aceitáveis.

Suplementação Alimentar; Aleitamento Materno; Recém-Nascido

\section{Referências}

1. Victora CG, Smith PG, Vaughan JP, Nobre LC, Lombardi C, Teixeira AM, et al. Evidence for protection by breast-feeding against infant deaths from infectious diseases in Brazil. Lancet 1987; 2:319-22.

2. World Health Organization/United Nations Children's Fund. Innocenti declaration on the protection, promotion and support breastfeeding, adopted by participants of WHO/UNICEF policymakers' meeting. Florence: World Health Organization/United Nations Children's Fund; 1990.

3. Fundo das Nações Unidas para a Infância. Manejo e promoção do aleitamento materno: curso de 18 horas para equipes de maternidades. Brasília: Fundo das Nações Unidas para a Infância; 1993.

4. Organização Mundial da Saúde/Fundo das Nações Unidas para a Infância/Ministério da Saúde. Guia de avaliação de Hospital Amigo da Criança. Brasília: Ministério da Saúde; 1999.

5. Pinto LM, Vitolo MR, Gírio LT, Leon MRAC, Zagari MCF, Farias NMF, et al. Aleitamento exclusivo em alojamento conjunto: avaliação da incidência e das causas do uso de fórmula. Rev Ciênc Méd PUCCAMP 1996; 5:63-8.

\section{Colaboradores}

C. A. B. Meirelles foi a responsável principal pela concepção geral do estudo, pela coleta e análise dos dados e pela redação do artigo. V. M. Fonseca participou da concepção geral do estudo, da análise dos dados e da redação do artigo. M. I. C. Oliveira e R. R. Mello contribuíram na redação do artigo e sua análise crítica. M. A. B Varela colaborou na coleta e análise dos dados.
6. Gagnon AJ, Leduc G, Waghorn K, Yang H, Platt RW. In-hospital formula supplementation of healthy breastfeeding newborns. J Hum Lact 2005; 21:397-404.

7. Carvalho M, Robertson S, Friedman A, Klaus M. Effect of frequent breastfeeding on early milk production and infant weight gain. Pediatrics 1983; 72:307-11.

8. Nantes MGD, Vinha VHP, Scochi CGS, Paula GA, Shimo AKK, Pela NTR. Tipos de mamilos em puérperas. Femina 1986; 14:692-5.

9. Sandre-Pereira G, Colares LGT, Carmo MGT, Soares EA. Conhecimentos maternos sobre amamentação entre puérperas inscritas em programa de pré-natal. Cad Saúde Pública 2000; 16:457-66.

10. Correa LG. Uso de suplemento em Hospital Amigo da Criança [Monografia de Graduação]. Duque de Caxias: Universidade do Grande Rio; 2005.

11. Connor EM, Sperling RS, Gelber R, Kiselev P, Scott G, O'Sullivan MJ, et al. Reduction of maternal-infant transmission of human immunodeficiency virus type 1 with zidovudine treatment. Pediatric AIDS Clinical Trials Group Protocol 076 Study Group. N Engl J Med. 1994; 331:1173-80. 
12. Oliveira MIC, Camacho LAB, Tedstone AE. A method for the evaluation of primary care unit's practice in the promotion, protection, and support of breastfeeding: results from the State of Rio de Janeiro, Brazil. J Hum Lact 2003; 19:365-73.

13. Lubchenco LO, Hansman C, Dressler M, Boyd E. Intrauterine growth as estimated from liveborn birth-weight data at 24 to 42 weeks of gestation. Pediatrics 1963; 32:793-800.

14. Alexander GR, Himes JH, Kaufman RB, Mor J, Kogan M. A United States national reference for fetal growth. Obstet Gynecol 1996; 87:163-8.

15. Ekström A, Nissen E. Duration of breastfeeding in Swedish primiparous and multiparous women. J Hum Lact 2003; 19:172-8.

16. Carvalhaes MA, Corrêa CRH. Identificação de dificuldades no início do aleitamento materno mediante aplicação de protocolo. J Pediatr (Rio J) 2003; 79:13-20.

17. Hopkinson JM, Schanler RJ, Garza C. Milk production by mothers of premature infants. Pediatrics 1988; 81:815-20.

18. Procianoy RS, Fernandes Filho PH, Sartori N. Influência de fatores neonatais sobre o aleitamento materno. J Pediatr (Rio J) 1982; 53:327-9.

19. Victora CG, Huttly SR, Barros FC, Vaughan JP. Cesarean section and duration of breast feeding among Brazilians. Arch Dis Child 1990; 65:632-4.
20. Perez-Escamilla R, Pollit E, Lönnerdal B, Dewey KG. Infant feeding policies in maternity wards and their effect on breastfeeding success: an analytical overview. Am J Public Health 1994; 84:89-97.

21. Berra S, Galván NK, Sabulsky J, Lafo BD, Gorgerino MC, Rajmil L, et al. Alimentación del recién nacido en el periodo de posparto inmediato. Rev Saúde Pública 2002; 36:661-9.

22. Righard L, Alade MO. Effect of delivery room routines on success of first breast-feed. Lancet 1990; 336:1105-7.

23. Programa Nacional de DST e AIDS, Secretaria de Vigilância em Saúde, Ministério da Saúde. Recomendações para profilaxia da transmissão vertical do HIV e terapia anti-retroviral em gestantes. Brasília: Ministério da Saúde; 2006.

24. Sanches MTC. Manejo clínico das disfunções orais na amamentação. J Pediatr 2004; 80(5 Suppl): S155-62.

25. Neifert TM, Lawrence R, Seacat J. Nipple confusion: toward a formal definition. J Pediatr 1995; 126:S125-9.

26. Rea MF. Substitutos do leite materno: passado e presente. Rev Saúde Pública 1990; 24:241-9.

27. Rezende MA, Sigaud CHS, Veríssimo MDLOR, Chiesa AM, Bertolozzi MR. O processo de comunicação na promoção do aleitamento materno. Rev Latinoam Enferm 2002; 10:234-8.

Recebido em 02/Ago/2007

Versão final reapresentada em 19/Fev/2008 Aprovado em 28/Fev/2008 